Melanie van der Heijden

Peter Pickkers

Geerten P. van Nieuw Amerongen

Victor W. M. van Hinsbergh

Martijn P. W. J. M. Bouw

Johannes G. van der Hoeven

A. B. Johan Groeneveld

\section{Circulating angiopoietin-2 levels in the course of septic shock: relation with fluid balance, pulmonary dysfunction and mortality}

Received: 24 February 2009

Accepted: 7 June 2009

Published online: 24 June 2009

(C) The Author(s) 2009. This article is published with open access at Springerlink.com

Electronic supplementary material The online version of this article (doi:10.1007/s00134-009-1560-y) contains supplementary material, which is available to authorized users.

M. van der Heijden - A. B. J. Groeneveld Department of Intensive Care,

VU University Medical Centre,

Amsterdam, The Netherlands

M. van der Heijden (®) · G. P. van Nieuw Amerongen - V. W. M. van Hinsbergh Department of Physiology, VU University Medical Centre, Van der Boechorststraat 7, 1081 BT Amsterdam, The Netherlands e-mail: m.vanderheijden@ vumc.nl

Tel.: +31-20-4441790

Fax: +31-20-4448255
P. Pickkers - M. P. W. J. M. Bouw · J. G. van der Hoeven

Department of Intensive Care,

Radboud University Medical Centre, Nijmegen, The Netherlands

Abstract Purpose: To investigate whether angiopoietin-2, von Willebrand factor (VWF) and angiopoietin1 relate to surrogate indicators of vascular permeability, pulmonary dysfunction and intensive care unit (ICU) mortality throughout the course of septic shock. Methods: In 50 consecutive mechanically ventilated septic shock patients, plasma angiopoietin-2, VWF and angiopoietin-1 levels and fluid balance, partial pressure of oxygen/inspiratory oxygen fraction and the oxygenation index as indicators of vascular permeability and pulmonary dysfunction, respectively, were measured until day 28. Results: Angiopoietin-2 positively related to the fluid balance and pulmonary dysfunction, was higher in non-survivors than in survivors and independently predicted non-survival throughout the course of septic shock. VWF inversely related to the fluid balance and pulmonary dysfunction throughout the course of septic shock, was comparable between survivors and non-survivors and predicted nonsurvival on day 0 only. Angiopoietin1 positively related to pulmonary dysfunction throughout the course, but did not differ between survivors and non-survivors. Conclusions: In contrast to VWF, plasma angiopoietin-2 positively relates to fluid balance, pulmonary dysfunction and mortality throughout the course of septic shock, in line with a suggested mediator role of the protein.

Keywords Angiopoietin . von Willebrand factor - Sepsis . Acute lung injury .

Vascular permeability

\section{Introduction}

Excessive and sustained activation of the endothelium resulting in increased vascular permeability is a crucial process during sepsis $[1,2]$. In the lung, the loss of plasma fluid into the interstitial space leads to pulmonary oedema, injury and dysfunction associated with acute lung injury (ALI) and acute respiratory distress syndrome (ARDS) $[1,3]$. Several clinical studies demonstrated that circulating levels of angiopoietin-2 (Ang-2) are elevated at the onset of critical illness in septic and non-septic patients with or at risk for ALI/ARDS, relate to vascular permeability and pulmonary dysfunction and are associated with intensive care unit (ICU) mortality [4-12]. It is not surprising that Ang-2 is a marker of those processes, since pre-formed Ang-2 can be released from the endothelial Weibel-Palade bodies during endothelial activation [13]. Another well-known marker of endothelial activation and dysfunction is von Willebrand factor (VWF), which is a major constituent of the Weibel-Palade body 
$[13,14]$. We previously showed that Ang-2 and VWF levels are simultaneously enhanced in the plasma of ALI/ ARDS or sepsis patients on admission to the ICU or onset of sepsis, suggesting that they are released together upon activation of the endothelium [12]. This may explain why both Ang-2 and VWF levels are associated with vascular permeability and pulmonary dysfunction $[4-6,8,11,12]$, even though VWF may not be directly involved in permeability $[15,16]$.

Experimental studies demonstrated that angiopoietin-1 (Ang-1) and Ang-2 bind to the endothelial Tie2 receptor [17-19]. Ang-1-induced activation of the Tie2 receptor results in vascular stabilisation [20-22]. Ang-2 provokes pulmonary inflammation and vascular leakage partly because of inhibition of the stabilising effect of Ang-1 by preventing Ang-1 from binding the Tie 2 receptor $[4,5,8$, $10-12,23,24]$. These experimental data together with clinical observations $[4,6,7,9,11,12]$ suggest that Ang2 is not only a marker, but also a direct mediator in the pathogenesis of vascular permeability, pulmonary injury and dysfunction. This idea would be further reinforced if the relation between Ang-2, vascular permeability and pulmonary dysfunction persists throughout the course of critical illness in a homogeneous population of septic shock patients, more than VWF and Ang-1 levels. Up till now, the course and clinical correlates of Ang-2 have not been studied, and the predictive value of changes in Ang2 during the course of septic shock for mortality is unknown. It has been hypothesised that the levels typically decrease in septic survivors and increase in septic patients that do not survive [5]. Only one study reported on the course of Ang-2 levels in multi-trauma patients [9], but clinical correlates were not studied.

The aims of our study were (1) to investigate whether Ang-2, VWF and Ang-1 relate to surrogate indicators of vascular permeability and pulmonary dysfunction throughout the course of septic shock and (2) to study the course of Ang-2, VWF and Ang-1 levels in survivors and non-survivors and their predictive value for ICU mortality. Therefore, we measured plasma levels of Ang-2, VWF and Ang-1, and recorded fluid balance as surrogate indicator of vascular permeability [25-28] and partial pressure of oxygen in arterial blood/inspiratory oxygen fraction $\left(P_{\mathrm{a}} \mathrm{O}_{2} / F_{\mathrm{i}} \mathrm{O}_{2}\right)$, oxygenation index [29], pulmonary compliance and ventilator-dependency [30] as surrogate indicators of pulmonary dysfunction, respectively, throughout the course of septic shock.

\section{Materials and methods}

The local ethics committee approved this observational study and waived the need for informed consent. Fifty consecutive mechanically ventilated critically ill patients with community or ICU-acquired septic shock admitted to the ICU of the Radboud University Medical Centre (Nijmegen, The Netherlands) between January 2005 and September 2007 were included within $12 \mathrm{~h}$ after meeting septic shock criteria. Sepsis was defined by two or more of the following: abnormal body temperature $(<36$ or $\left.>38.5^{\circ} \mathrm{C}\right)$, tachycardia $(>90 / \mathrm{min})$, tachypnea $[>20 / \mathrm{min}$ or partial pressure of $\mathrm{CO}_{2}$ in arterial blood $\left(\mathrm{P}_{\mathrm{a}} \mathrm{CO}_{2}\right)$ $<32 \mathrm{mmHg}$ ), abnormal white blood cell counts $(<4$ or $\left.>12 \times 10^{\circ} / 1\right)$ and a microbiologically proven or clinically evident source of infection [29]. Septic shock was defined as sepsis complicated by acute circulatory failure characterised by persistent arterial hypotension unexplained by other causes [31]. Hypotension was defined by systolic arterial pressure $<90 \mathrm{mmHg}$ or mean arterial pressure (MAP) $<70 \mathrm{mmHg}$ for at least $1 \mathrm{~h}$ despite adequate fluid resuscitation or requirement of vasopressor support to maintain MAP [31]. ICU-acquired sepsis was defined as sepsis developing after 2 days in the ICU. The origin of septic shock was defined by clinical signs and symptoms and positive local and/or blood cultures. Patients were pressure-controlled or volume-controlled (after surgery) ventilated with a Galileo (Hamilton Medical AG; Rhäzüns, Switserland) or a Servo ventilator (Maquet, Inc.; Bridgewater, NJ). Patients with ALI/ARDS were pressurecontrolled ventilated with a tidal volume $\left(V_{\mathrm{t}}\right)$ aiming not to exceed $8 \mathrm{ml} / \mathrm{kg}$ and resulting in an end-tidal $\mathrm{CO}_{2}$ concentration between 4 and 5\%, using an $\mathrm{O}_{2}$-air mixture with a $F_{\mathrm{i}} \mathrm{O}_{2}$ of $40 \%$ and a positive end-expiratory pressure (PEEP) of $5 \mathrm{cmH}_{2} \mathrm{O}$ (inspiration:expiration 1:2) or more, when needed, guided by $P_{\mathrm{a}} \mathrm{O}_{2}(>60 \mathrm{mmHg})$. Patients were followed until death or discharge from the hospital. The 28-day ICU mortality, total ICU mortality and hospital mortality were recorded. Patients were treated by intensive care physicians not involved in the study according to institutional guidelines. Platelets were infused when the platelet count had dropped below 10-20 × 109/1, possibly as a result of diffuse intravascular coagulation, and when there were foci with a bleeding tendency.

On the inclusion day (day 0), demographics and clinical data were recorded including the Acute Physiology and Chronic Health Evaluation (APACHE) II score. The number of failing organs was scored. On day 0 , day $7 \pm 1$ days, day $14 \pm 2$ days and day $28 \pm 3$ days, arterial blood samples were obtained for determinations of $\mathrm{pH}, P_{\mathrm{a}} \mathrm{O}_{2}$ and $P_{\mathrm{a}} \mathrm{CO}_{2}$, white blood cell counts and creatinine. The respiratory frequency, tidal volume $\left(V_{\mathrm{t}}\right), F_{\mathrm{i}} \mathrm{O}_{2}$, plateau pressure $\left(P_{\text {plat }}\right)$, mean airway pressure $\left(P_{\text {mean }}\right)$ and PEEP were taken from the ventilator. The oxygenation index, which is a measure of oxygenation impairment for the intensity of mechanical ventilation, was calculated from $\left(P_{\text {mean }} \times F_{\mathrm{i}} \mathrm{O}_{2} \times 100\right) /$ $P_{\mathrm{a}} \mathrm{O}_{2}$ [29]. Total respiratory dynamic compliance was calculated from $V_{\mathrm{t}} /$ (plateau pressure-PEEP). Ventilatorfree days were defined as follows: ventilator-free days $=0$ if the patient dies before ICU day 28; ventilator-free days $=28-x$ if the patient is successfully 
weaned from mechanical ventilation within 28 days, where $x$ is the number of days spent on mechanical ventilation; ventilator-free days $=0$ if the patient requires mechanical ventilation for 28 days or more [30]. Days at which a patient was ventilator-dependent were recorded. ALI and ARDS were characterised by $P_{\mathrm{a}} \mathrm{O}_{2} /$ $F_{\mathrm{i}} \mathrm{O}_{2} \leq 300$ or $\leq 200 \mathrm{mmHg}$, respectively, and absence of clinical evidence of left atrial hypertension and congestive heart failure, according to the American European Consensus Criteria [32]. Vasopressor/inotropic treatment and body temperature were recorded. The fluid balance was calculated by dividing total fluid input minus total fluid output by the pre-ICU patient weight in kilograms [25-28]. Circulating Ang-2, VWF and Ang-1 were measured as described in the electronic supplementary material (ESM). The statistical analysis is described in the ESM.

\section{Results}

Patient characteristics

Patients were grouped as ICU survivor or non-survivor (Table 1, ESM Table 1). All ICU non-survivors, except one (death on day 68), died before ICU day 28. Five ICU survivors died in the hospital at a median 24 (range 2-60) days after discharge from the ICU. ICU survivors and non-survivors were comparable with respect to baseline demographics and with respect to clinical characteristics, except for APACHE II score and hospital stay, which were higher and shorter, respectively, in non-survivors compared to survivors. Twenty-two survivors and 14 nonsurvivors suffered from ALI/ARDS (Table 1). Haemodynamic, biochemical and respiratory characteristics are reported in the ESM.

Angiopoietin and VWF levels, fluid balance and pulmonary dysfunction

Patients had higher Ang-2 and VWF levels on day 0 [median (interquartile range) $2,350(1,400-4,280) \mathrm{pg} / \mathrm{ml}$ and 289 (166-411)\% of reference, respectively] than controls [113 (27-287) pg/ml, $P<0.001$ and 93 (87$113) \%$ of reference, $P<0.001$, respectively]. Patients had lower Ang-1 levels on day $0[330(114-1,020) \mathrm{pg} /$ $\mathrm{ml}$ than controls [2,407 (969-3,730) pg/ml, $P<0.001]$. Ang-2 levels related positively to the fluid balance throughout the course of septic shock after exclusion of one non-survivor who had extremely high Ang-2 levels ( $\beta=0.20, P=0.043$ in GEE, $r=0.30, P=0.005$ ). Furthermore, Ang-2 levels related to indicators of pulmonary dysfunction throughout the course of septic shock, as demonstrated by an inverse relation with the
$P_{\mathrm{a}} \mathrm{O}_{2} / F_{\mathrm{i}} \mathrm{O}_{2}$ ratio $(\beta=-0.25, P<0.001$ in GEE; $r=$ $-0.25, P=0.012)$, a positive relation with the oxygenation index $(\beta=0.37, P<0.001$ in GEE; $r=0.26$, $P=0.017)$, a weak inverse relation with the compliance ( $\beta=-0.09, P=0.004$ in GEE; $r=-0.05, P=0.645$ ) and a positive relation with ventilator-dependency $(P=0.043$ in GEE). In contrast, VWF inversely related to the fluid balance $(\beta=-0.21, P=0.045$ in GEE; $r=-0.27, P=0.014)$, positively related to the compliance $(\beta=0.21, \quad P=0.011$ in GEE; $r=0.17$, $P=0.134)$ and did not relate to the other respiratory variables throughout the course of septic shock. Ang-1 levels did not relate to the fluid balance, but related to indicators of pulmonary dysfunction throughout the course of septic shock, as demonstrated by an inverse relation with the $P_{\mathrm{a}} \mathrm{O}_{2} / F_{\mathrm{i}} \mathrm{O}_{2}$ ratio $(\beta=-0.26$, $P=0.022$ in GEE; $r=-0.21, P=0.034)$ and a positive relation with the oxygenation index $(\beta=0.37$, $P=0.005$ in GEE; $r=0.28, P=0.014$ ) throughout the course of septic shock. The fluid balance related to indicators of pulmonary dysfunction throughout the course of septic shock, as demonstrated by an inverse relation with the $P_{\mathrm{a}} \mathrm{O}_{2} / F_{\mathrm{i}} \mathrm{O}_{2}$ ratio $(\beta=-0.24, P<0.001$ in GEE; $r=-0.29, P=0.006)$, a positive relation with the oxygenation index $(\beta=0.22, P=0.006$ in GEE; $r=0.22, P=0.068)$ and an inverse relation with the compliance $(\beta=-0.26, P=0.006$ in GEE; $r=-0.29$, $P=0.014)$.

The course of the angiopoietin and VWF levels in survivors and non-survivors

Ang-2 levels were higher in non-survivors than in survivors throughout the course of septic shock (Fig. 1). Ang-2 levels decreased in survivors from day 0 to the last ICU day, while they did not change in non-survivors (Figs. 1, 2 ). The course of VWF levels differed between survivors and non-survivors (Fig. 1), since VWF levels increased in survivors from day 0 to the last ICU day, while they did not change in non-survivors (Fig. 2). Ang-2 levels related to VWF levels on day 0 , but the relation was lost throughout the course of septic shock (Fig. 3). Ang-1 levels were comparable between survivors and non-survivors (Fig. 1) and did not change from day 0 to the last ICU day (Fig. 2). The predictive value of angiopoietin and VWF levels for ICU mortality is described in the ESM.

\section{Discussion}

This study demonstrates that circulating Ang-2 positively relates to the fluid balance, pulmonary dysfunction and 
Table 1 Demographic and clinical characteristics of survivors and non-survivors

\begin{tabular}{|c|c|c|c|}
\hline & $\begin{array}{l}\text { Survivors } \\
(n=35)\end{array}$ & $\begin{array}{l}\text { Non-survivors } \\
(n=15)\end{array}$ & $P$ value \\
\hline Age, year & $68(21-87)$ & $65(30-102)$ & 0.427 \\
\hline Sex, male & $21(60)$ & $13(87)$ & 0.099 \\
\hline Surgery prior to sepsis & 17 (49) & $9(60)$ & 0.459 \\
\hline Source of sepsis & & & 0.529 \\
\hline Pulmonary & $15(43)$ & $9(60)$ & \\
\hline Abdominal & $15(43)$ & $5(33)$ & \\
\hline Urogenital & $1(3)$ & 0 & \\
\hline Pressure sores & $1(3)$ & 0 & \\
\hline Unknown & $5(14)$ & $2(13)$ & \\
\hline Culture results & & & 0.241 \\
\hline Blood gram - & $2(6)$ & $1(7)$ & \\
\hline Blood gram + & 0 & $2(13)$ & \\
\hline Local $^{\mathrm{a}}$ gram - & $5(14)$ & $3(9)$ & \\
\hline Local $^{\mathrm{a}}$ gram + & $7(20)$ & $1(7)$ & \\
\hline Leukocytes day $0, \times 10^{9} / 1$ & $11.9(2.8-86.0)$ & $2.7(0.4-74.8)$ & $0.661,0.209$ \\
\hline Leukocytes day $7, \times 10^{9} / 1$ & $15.6(5.6-54.5)$ & $11.3(1.7-243.1)$ & \\
\hline Leukocytes day $14, \times 10^{9} / 1$ & $12.8(7.5-33.8)$ & $18.8(4.1-101.0)$ & \\
\hline Temperature day $0,{ }^{\circ} \mathrm{C}$ & $37.7(34.4-41.2)$ & $38.0(34.3-40.4)$ & $0.288,0.600$ \\
\hline Temperature day $7,{ }^{\circ} \mathrm{C}$ & $37.5(35.6-39.8)$ & $38.2(34.0-40.0)$ & \\
\hline Temperature day $14,{ }^{\circ} \mathrm{C}$ & $38.1(35.5-39.3)$ & $38.1(37.2-39.9)$ & \\
\hline Number of failing organs & $2(1-6)$ & $2(1-7)$ & 0.425 \\
\hline Severity of lung injury & & & 0.869 \\
\hline Non-ALI & $3(9)$ & $1(7)$ & \\
\hline ALI & $9(26)$ & $3(9)$ & \\
\hline ARDS & $23(66)$ & $11(73)$ & \\
\hline ICU-acquired sepsis & $4(12)$ & $1(6)$ & 1.000 \\
\hline APACHE II score & $20.5(9.0-34.0)$ & $22.0(18.0-53.0)$ & 0.014 \\
\hline Ventilator-free days & $14(0-27)$ & 0 & $<0.001$ \\
\hline ICU stay, days & $13(2-86)$ & $6(1-68)$ & 0.056 \\
\hline Hospital stay, days & $53(6-146)$ & $10(1-83)$ & 0.002 \\
\hline
\end{tabular}

Variables are expressed as median (range) or as number of patients (percentage) with $P$ value (survivor vs. non-survivor and interaction between day and survivor vs. non-survivor)

Non-ALI non-acute lung injury defined as partial pressure of $\mathrm{O}_{2}$ in arterial blood/inspiratory $\mathrm{O}_{2}$ fraction $\left(P_{\mathrm{a}} \mathrm{O}_{2} / F_{\mathrm{i}} \mathrm{O}_{2}\right)$ at day 0 $>300 \mathrm{mmHg}, A L I$ defined as $P_{\mathrm{a}} \mathrm{O}_{2} / F_{\mathrm{i}} \mathrm{O}_{2}$ at day $0>200$ and

mortality throughout the course of septic shock. This is in line with the suggested mediator role of Ang-2 in those patients. In contrast, VWF was not positively related to the fluid balance and pulmonary dysfunction and predicted mortality only at the day of ICU admission, suggesting that VWF plays a marker role only in the early phase of septic shock. Indeed, circulating VWF and Ang2 were related at day 0 , but not throughout the course of septic shock.

Experimental data $[5,19,23]$ together with several observations in patient studies [4-9, 11, 12, 25] suggest that Ang-2 is a mediator in the pathogenesis of pulmonary vascular permeability and injury. This hypothesis is reinforced by several aspects of the present study. First, the relation between circulating Ang-2 and the fluid balance or pulmonary dysfunction persisted throughout the course of septic shock. Second, this longitudinal relation was Ang-2-specific, since VWF, which is also considered a marker of endothelial activation and dysfunction, did not reflect a positive fluid balance and pulmonary $\leq 300 \mathrm{mmHg}, A R D S$ acute respiratory distress syndrome defined as $P_{\mathrm{a}} \mathrm{O}_{2} / F_{\mathrm{i}} \mathrm{O}_{2}$ at day $0 \leq 200 \mathrm{mmHg}, I C U$ intensive care unit, APACHE Acute Physiology And Chronic Health Evaluation

${ }^{a}$ Local when blood-culture negative

dysfunction. Third, the relation was observed within a relatively homogeneous group of septic patients with high Ang-2 levels and severe vascular permeability and pulmonary dysfunction, while previous studies focused on heterogeneous populations $[4-7,10-12]$. In those heterogeneous populations, Ang-2, vascular permeability and pulmonary dysfunction may be related due to a group effect, because non-septic patients have low Ang-2 levels and moderate vascular permeability or pulmonary dysfunction, while septic patients have high Ang-2 levels and severe vascular permeability or pulmonary dysfunction. Nevertheless, in vivo Ang-2 blocking studies are necessary to formally confirm the mediator role of Ang-2.

Ang-2 levels at day 0 and throughout the course had independent predictive value for 28 day survival, in accordance with a previous study [10], since patients with Ang-2 levels $<3,066 \mathrm{pg} / \mathrm{ml}$ had longer survival duration than patients with Ang-2 levels $\geq 3,066 \mathrm{pg} / \mathrm{ml}$, independent of the APACHE II score. Nevertheless, these cutoff values were selected post-hoc and should be validated 
Fig. 1 The course of angiopoietin and VWF levels in survivors and non-survivors. Median and interquartile range of a circulating angiopoietin-2 (Ang-2; pg/ml), b von Willebrand factor (VWF; \% of reference) and $\mathbf{c}$ angiopoietin-1 (Ang-1) levels (pg/ml) in plasma of intensive care unit (ICU) survivors (open circle) and ICU nonsurvivors (filled circle) on day 0,1, 2, 4 and 7. Days 14, 21 and 28 are not shown because of low numbers, but are included in the analysis. a Ang-2 levels tended to be higher in non-survivors than in survivors throughout the course of septic shock $(P=0.199$ for survivor vs. non-survivor and $P=0.028$ for interaction between day and survivor vs. non-survivor). One non-survivor had extremely high Ang-2 levels: day $0125,100 \mathrm{pg} / \mathrm{ml}$; day 1 $149,000 \mathrm{pg} / \mathrm{ml}$; day $2114,300 \mathrm{pg} / \mathrm{ml}$; day $4115,550 \mathrm{pg} / \mathrm{ml}$ and day $7116,800 \mathrm{pg} / \mathrm{ml}$. Therefore, the upper limit of interquartile range at day 7 is $88,263 \mathrm{pg} / \mathrm{ml}$. Analysis without this non-survivor yields a $P=0.039$ for survivor versus non-survivor and $P=0.016$ for the interaction between day and survivor versus non-survivor. $\mathbf{b}$ VWF levels did not differ between survivors and non-survivors throughout the course of septic shock $(P=0.545$ for survivor vs. non-survivor and $P=0.218$ for interaction between day and survivor vs. non-survivor). c Ang-1 levels did not differ between survivors and non-survivors throughout the course of septic shock $(P=0.189$ for survivor vs. non-survivor and $P=0.070$ for interaction between day and survivor vs. non-survivor)

prospectively. It has been proposed previously that circulating Ang-2 levels may better predict mortality of critically ill patients than the APACHE II score [7, 11]. For instance, Siner et al. [11] reported that there were no deaths among patients with Ang-2 levels less than $10,000 \mathrm{pg} / \mathrm{ml}$, despite a predicted mortality of $16 \%$ based on the median APACHE II score. Indeed, the combination of Ang-2 and the APACHE II score had more predictive value than the APACHE II score alone. In our patient group, we did not observe a sharp increase in Ang-2 levels towards the day of death as was described for one patient by Parikh et al. [5]. Ang-2 was continuously higher in non-survivors compared to survivors. Interestingly, Ang-2 levels in survivors, which were already lower throughout the course, decreased from day 0 to the last ICU day. In this light, Ang-2 levels above a certain threshold may mediate the development of endothelial activation and dysfunction, associated with pulmonary dysfunction and mortality. Surprisingly, Ang-1 levels also positively related to pulmonary dysfunction. Nevertheless, Ang-1 levels in our septic shock patients were still much lower than Ang-1 levels in healthy controls according to the literature $[10,12]$, suggesting that the low Ang-1 levels did not offset the effects of high Ang-2 levels. Indeed, the Ang-2/Ang-1 ratio did not relate to the fluid balance or pulmonary dysfunction and mortality (data not shown).

VWF levels related inversely to the fluid balance and positively to the pulmonary compliance throughout the course of septic shock. This finding is in accordance with studies that did not find a predictive value for the development of ALI/ARDS [33, 34], while other studies reported that VWF levels at the onset of critical illness predicted the development of ALI/ARDS and related to pulmonary permeability $[12,35]$. It is unclear how these
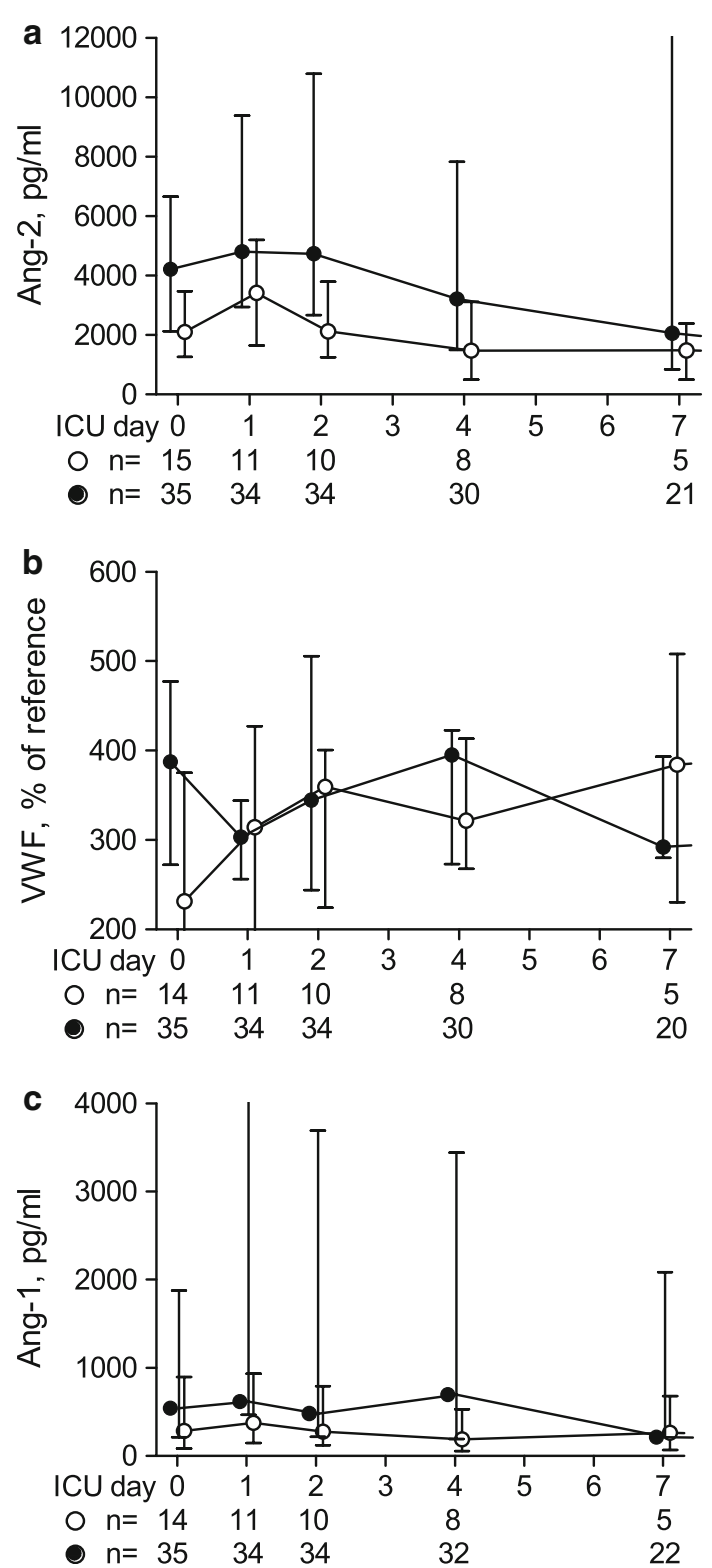

differences can be explained, but the predictive value of VWF might depend on the time at which the samples were taken during the course of sepsis or ALI/ARDS. Indeed, VWF had predictive value for mortality early in the course of sepsis and ALI/ARDS (day 0), as reported by most [15, 16, 33, 35], but not all prior studies [36], but lost its predictive value on the subsequent days. Although VWF may thus have an important early prognostic role, the data do not suggest a direct pathogenetic role of the factor, in contrast to Ang-2.

In our study, increased Ang-2 and VWF levels were related on day 0 as reported before $[8,12]$, suggesting that they were released together from the endothelial WeibelPalade body, but not on the subsequent days. There are several explanations for the observed loss of relation 

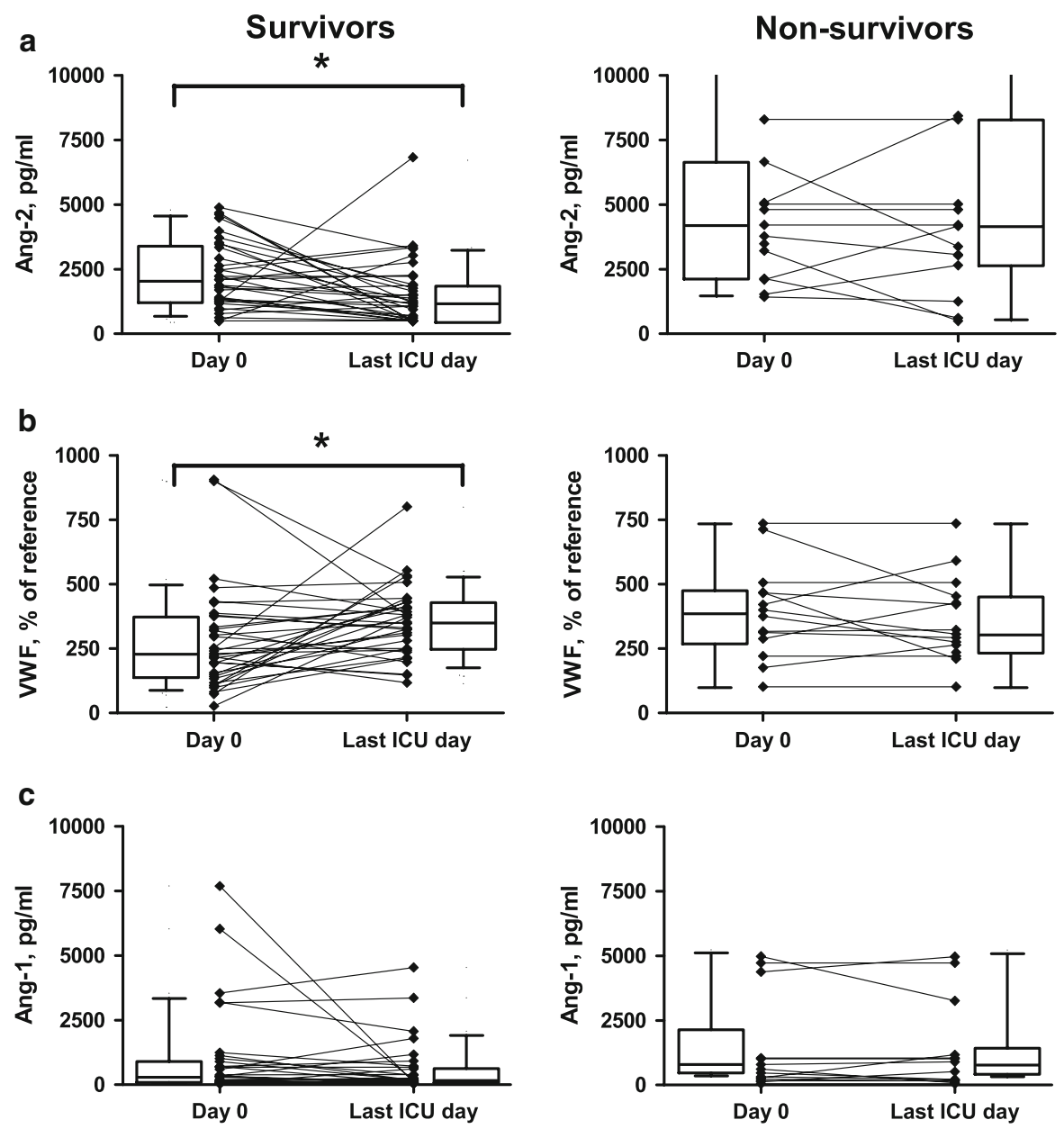

Fig. 2 Angiopoietin and VWF levels on day 0 and the last ICU day in survivors and non-survivors. Individual levels and box plot of a circulating angiopoietin-2 (Ang-2; pg/ml), b von Willebrand factor (VWF; \% of reference) and c angiopoietin-1 (Ang-1) levels (pg/ml) on day 0 and the last intensive care unit (ICU) day in ICU survivors and ICU non-survivors. Values in boxes are at 25th percentile, median and 75th percentile; whiskers are 10th and 90th percentile. a Ang-2 levels decreased from day 0 to the last ICU day in survivors ( $P P=0.0033, n=35$ pairs), while they did not change in nonsurvivors $(P=0.3910, n=15$ pairs $)$. Data not shown: Day 0

between Ang-2 and VWF levels. First, the synthesis and subsequent storage of Ang-2 and VWF in de Weibel-Palade bodies may become differently regulated throughout the course of disease, so that the ratio between Ang-2 and VWF changed. Second, Ang-2 may not only be released from the Weibel-Palade body, but also by constitutive secretion from the cytoplasm. Indeed, Ang-2 is uniformly distributed in the cytoplasm of endothelial cells in the absence of VWF [13]. Third, the binding or consumption of VWF may be increased in more severely ill patients, for example, during diffuse intravascular coagulation.

Our study has several limitations. First, the relation between Ang-2 and a positive fluid balance or pulmonary dysfunction was relatively modest $(r=0.25-0.30)$,

survivor: $25,090 \mathrm{pg} / \mathrm{ml}$; day 0 non-survivor: 17,980 and 125,100 pg/ $\mathrm{ml}$; last ICU day non-survivor: 12,050 and $116,800 \mathrm{pg} / \mathrm{ml}$; 90th percentile of non-survivors day $0: 60,828 \mathrm{pg} / \mathrm{ml}$; 90 th percentile of non-survivors last ICU day: 53,950 pg/ml. b VWF levels increased from day 0 to the last ICU day in survivors $(* P=0.0057, n=35$ pairs $)$ and did not change in non-survivors $(P=0.5070, n=14$ pairs). c Ang-1 levels did not differ between day 0 and the last ICU day in survivors $(P=0.1044, n=35$ pairs $)$ or non-survivors $(P=0.7612, n=14$ pairs $)$

suggesting that the positive fluid balance and pulmonary dysfunction are only partly due to increased Ang-2 levels. Indeed, it has been reported that Ang-2 sensitizes the endothelium to inflammatory stimuli rather than acting alone in the vascular system [37]. Second, it was not feasible to measure the pulmonary vascular permeability in a more direct manner with the pulmonary leak index method as we did in our previous study [12]. Instead, we studied the relation with fluid balance as surrogate indicator of vascular permeability. The data suggest that differences and changes in fluid balance were not confounded by differences and changes in renal function as can be judged, albeit imperfectly, from serum creatinine levels. Therefore, changes in the fluid balance are likely explained, at least in part, by 


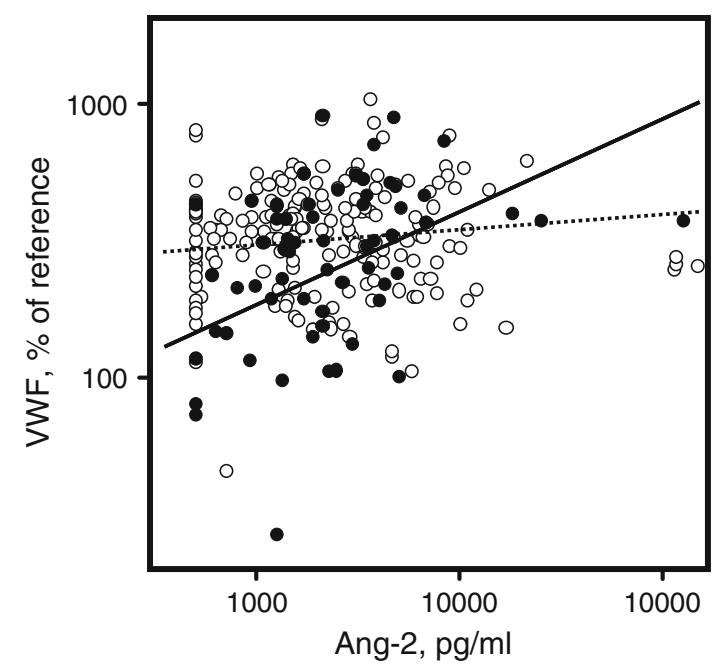

Fig. 3 The relation between Ang-2 and VWF levels. Angiopoietin2 (Ang-2) levels $(\mathrm{pg} / \mathrm{ml})$ positively related to von Willebrand factor (VWF) levels (\% of reference) at day 0 (filled circle, $r=0.45$, $P=0.001$, black line). Ang-2 levels throughout the course of septic shock did not relate to VWF levels (open circle day 1, day 2 , day 4 , day 7 , day 14 , day 21 and day $28, r=0.10, P=0.1190$, dotted line)

changes in vascular permeability, even though not reflecting pulmonary vascular permeability directly. However, it is likely that the systemic and pulmonary vascular endothelium are simultaneously activated, since both are exposed to circulating inflammatory mediators. Furthermore, the fluid balance related to the $P_{\mathrm{a}} \mathrm{O}_{2} / F_{\mathrm{i}} \mathrm{O}_{2}$ ratio and the oxygenation index, and we confirmed that a positive fluid balance is related to a worse outcome in ALI and septic shock [26, 27], suggesting that a positive fluid balance includes pulmonary injury-induced oedema. Third, the plasma levels of Ang-2, VWF and Ang-1 and the difference in Ang-2 levels between survivors and non-survivors may have been underestimated due to fluid resuscitation and plasma dilution. This indicates that the difference in plasma levels of the measured proteins may be even greater and the relation with the fluid balance and pulmonary dysfunction even stronger. Fourth, in multivariate analysis, APACHE II score and Ang-2, but not fluid balance, $P_{\mathrm{a}} \mathrm{O}_{2} / F_{\mathrm{i}} \mathrm{O}_{2}$ ratio, oxygenation index, PEEP and Ang- 1 at day 0 remained significantly predictive factors of ICU mortality. Nevertheless, the value of this analysis is limited in our relatively small group of 50 patients.

In conclusion, in contrast to VWF levels, increased Ang-2 levels relate to a positive fluid balance and pulmonary dysfunction throughout the course of septic shock. While VWF levels only predict ICU mortality early in the course of septic shock, Ang-2 levels do so throughout the course. Pharmacological blockade of Ang-2 during the course of septic shock might reduce the degree of vascular permeability and pulmonary dysfunction and should be pursued in future studies for outcome benefits.

Acknowledgments We thank Jan van Bezu BSc., VU University Medical Centre, Amsterdam, The Netherlands, for excellent technical assistance. GpvNA was supported by The Netherlands Heart Foundation (The Hague, grant 2003T032). MvdH was supported by the European Society of Intensive Care Medicine (ECCRN Levi Montalcini Biomedical Sciences Award 2007). The funding sources had no involvement in the study design, the collection, analysis and interpretation of data, the writing of the report or the decision to submit the paper for publication.

Open Access This article is distributed under the terms of the Creative Commons Attribution Noncommercial License which permits any noncommercial use, distribution, and reproduction in any medium, provided the original author(s) and source are credited.

\section{References}

1. Hotchkiss RS, Karl IE (2003) The pathophysiology and treatment of sepsis. N Engl J Med 348:138-150

2. Aird WC (2003) The role of the endothelium in severe sepsis and multiple organ dysfunction syndrome. Blood 101:3765-3777

3. Groeneveld ABJ (2002) Vascular pharmacology of acute lung injury and acute respiratory distress syndrome. Vascul Pharmacol 39:247-256

4. Orfanos SE, Kotanidou A, Glynos C, Athanasiou C, Tsigkos S, Dimopoulou I, Sotiropoulou C, Zakynthinos S, Armaganidis A, Papapetropoulos A, Roussos C (2006) Angiopoietin-2 is increased in severe sepsis: correlation with inflammatory mediators. Crit Care Med 35:199-206
5. Parikh SM, Mammoto T, Schultz A, Yuan HT, Christiani D, Karumanchi SA, Sukhatme VP (2006) Excess circulating angiopoietin-2 may contribute to pulmonary vascular leak in sepsis in humans. PLoS Med 3:356370

6. Giuliano JS Jr, Lahni PM, Harmon K, Wong HR, Doughty LA, Carcillo JA, Zingarelli B, Sukhatme VP, Parikh SM, Wheeler DS (2007) Admission angiopoietin levels in children with septic shock. Shock 28:650-654

7. Gallagher DC, Parikh SM, Balonov K, Miller A, Gautam S, Talmor D, Sukhatme VP (2008) Circulating angiopoietin 2 correlates with mortality in a surgical population with acute lung injury/adult respiratory distress syndrome. Shock 29:656-661
8. Ganter MT, Cohen MJ, Brohi K, Chesebro BB, Staudenmayer KL, Rahn P, Christiaans SC, Bir ND, Pittet JF (2008) Angiopoietin-2, marker and mediator of endothelial activation with prognostic significance early after trauma? Ann Surg 247:320-326

9. Giamarellos-Bourboulis EJ, Kanellakopoulou K, Pelekanou A, Tsaganos T, Kotzampassi K (2008) Kinetics of angiopoietin-2 in serum of multi-trauma patients: correlation with patient severity. Cytokine 44:310-313

10. Kumpers P, Lukasz A, David S, Horn R, Hafer C, Faulhaber-Walter R, Fliser D, Haller H, Kielstein JT (2008) Excess circulating angiopoietin-2 is a strong predictor of mortality in critically ill medical patients. Crit Care 12:R147 
11. Siner JM, Bhandari V, Engle KM, Elias JA, Siegel MD (2009) Elevated serum angiopoietin-2 levels are associated with increased mortality in sepsis. Shock 31:348-353

12. Van der Heijden M, Van Nieuw Amerongen GP, Koolwijk P, Van Hinsbergh VWM, Groeneveld ABJ (2008) Angiopoietin-2, permeability oedema, occurrence and severity of ALI/ARDS in septic and non-septic critically ill patients. Thorax 63:903909

13. Fiedler U, Scharpfenecker M, Koidl S, Hegen A, Grunow V, Schmidt JM, Kriz W, Thurston G, Augustin HG (2004) The Tie-2 ligand angiopoietin-2 is stored in and rapidly released upon stimulation from endothelial cell Weibel-Palade bodies. Blood 103:4150-4156

14. Rondaij MG, Bierings R, Kragt A, Van Mourik JA, Voorberg J (2006) Dynamics and plasticity of WeibelPalade bodies in endothelial cells. Arterioscler Thromb Vasc Biol 26:1002-1007

15. Ware LB, Conner ER, Matthay MA (2001) von Willebrand factor antigen is an independent marker of poor outcome in patients with early acute lung injury. Crit Care Med 29:2325-2331

16. Ware LB, Eisner MD, Thompson BT, Parsons PE, Matthay MA (2004)

Significance of von Willebrand factor in septic and nonseptic patients with acute lung injury. Am J Respir Crit Care Med 170:766-772

17. Eklund L, Olsen BR (2006) Tie receptors and their angiopoietin ligands are context-dependent regulators of vascular remodeling. Exp Cell Res 312:630-641

18. Maisonpierre PC, Suri C, Jones PF, Bartunkova S, Wiegand SJ, Radziejewski C, Compton D, McClain J, Aldrich TH, Papadopoulos N, Daly TJ, Davis S, Sato TN, Yancopoulos GD (1997) Angiopoietin-2, a natural antagonist for Tie2 that disrupts in vivo angiogenesis. Science 277:55-60

19. Scharpfenecker M, Fiedler U, Reiss Y, Augustin HG (2005) The Tie-2 ligand angiopoietin-2 destabilizes quiescent endothelium through an internal autocrine loop mechanism. J Cell Sci 118:771-780

20. Mammoto T, Parikh SM, Mammoto A, Gallagher D, Chan B, Mostoslavsky G, Ingber DE, Sukhatme VP (2007)

Angiopoietin-1 requires p190 RhoGAP to protect against vascular leakage in vivo. J Biol Chem 282:23910-23918
21. McCarter SD, Mei SH, Lai PF, Zhang QW, Parker CH, Suen RS, Hood RD, Zhao YD, Deng Y, Han RN, Dumont DJ, Stewart DJ (2007) Cell-based angiopoietin-1 gene therapy for acute lung injury. Am J Respir Crit Care Med 175:1014-1026

22. Witzenbichler B, Westermann D, Knueppel S, Schultheiss HP, Tschope C (2005) Protective role of angiopoietin-1 in endotoxic shock. Circulation 111:97105

23. Bhandari V, Choo-Wing R, Lee CG, Zhu Z, Nedrelow JH, Chupp GL, Zhang X, Matthay MA, Ware LB, Homer RJ, Lee PJ, Geick A, de Fougerolles AR, Elias JA (2006) Hyperoxia causes angiopoietin 2-mediated acute lung injury and necrotic cell death. Nat Med 12:1286-1293

24. Van der Heijden M, Van Nieuw Amerongen GP, Chedamni S, Van Hinsbergh VWM, Groeneveld ABJ (2009) The angiopoietin-Tie 2 system as a therapeutic target in sepsis and acute lung injury. Expert Opin Ther Targets $13: 39-53$

25. Giuliano JS Jr, Lahni PM, Bigham MT, Manning PB, Nelson DP, Wong HR, Wheeler DS (2008) Plasma angiopoietin-2 levels increase in children following cardiopulmonary bypass. Intensive Care Med 34:18511857

26. Sakr Y, Vincent JL, Reinhart K, Groeneveld J, Michalopoulos A, Sprung CL, Artigas A, Ranieri VM (2005) High tidal volume and positive fluid balance are associated with worse outcome in acute lung injury. Chest 128:3098-3108

27. Alsous F, Khamiees M, DeGirolamo A, Moateng-Adjepong Y, Manthous CA (2000) Negative fluid balance predicts survival in patients with septic shock: a retrospective pilot study. Chest 117:1749-1754

28. Pickkers P, Sprong T, Eijk L, Van der Hoeven JG, Smits P, Deuren M (2005) Vascular endothelial growth factor is increased during the first $48 \mathrm{~h}$ of human septic shock and correlates with vascular permeability. Shock 24:508512

29. Seeley E, McAuley DF, Eisner M, Miletin M, Matthay MA, Kallet RH (2008) Predictors of mortality in acute lung injury during the era of lung protective ventilation. Thorax 63:994998
30. Schoenfeld DA, Bernard GR (2002) Statistical evaluation of ventilator-free days as an efficacy measure in clinical trails of treatments for acute respiratory distress syndrome. Crit Care Med 30:1772-1777

31. Levy MM, Fink MP, Marshall JC, Abraham E, Angus D, Cook D, Cohen J, Opal SM, Vincent JL, Ramsay G (2003) $2001 \mathrm{SCCM} / \mathrm{ESICM} / \mathrm{ACCP} /$ ATS/SIS International Sepsis Definitions Conference. Crit Care Med 31:1250-1256

32. Bernard GR, Artigas A, Brigham KL, Carlet J, Falke K, Hudson L, Lamy M, LeGall JR, Morris A, Spragg R (1994) Report of the American-European consensus conference on ARDS: definitions, mechanisms, relevant outcomes and clinical trial coordination. The Consensus Committee. Intensive Care Med 20:225-232

33. Kayal S, Jais JP, Aguini N, Chaudiere J, Labrousse J (1998) Elevated circulating E-selectin, intercellular adhesion molecule 1, and von Willebrand factor in patients with severe infection. Am J Respir Crit Care Med 157:776-784

34. Moss M, Ackerson L, Gillespie MK, Moore FA, Moore EE, Parsons PE (1995) von Willebrand factor antigen levels are not predictive for the adult respiratory distress syndrome. Am J Respir Crit Care Med 151:15-20

35. Rubin DB, Wiener-Kronish JP, Murray JF, Green DR, Turner J, Luce JM, Montgomery AB, Marks JD, Matthay MA (1990) Elevated von Willebrand factor antigen is an early plasma predictor of acute lung injury in nonpulmonary sepsis syndrome. J Clin Invest 86:474-480

36. Sabharwal AK, Bajaj SP, Ameri A, Tricomi SM, Hyers TM, Dahms TE, Taylor FB Jr, Bajaj MS (1995) Tissue factor pathway inhibitor and von Willebrand factor antigen levels in adult respiratory distress syndrome and in a primate model of sepsis. Am $\mathbf{J}$ Respir Crit Care Med 151:758-767

37. Fiedler U, Reiss Y, Scharpfenecker M, Grunow V, Koidl S, Thurston G, Gale NW, Witzenrath M, Rosseau S, Suttorp N, Sobke A, Herrmann M, Preissner KT, Vajkoczy P, Augustin HG (2006) Angiopoietin-2 sensitizes endothelial cells to TNF-alpha and has a crucial role in the induction of inflammation. Nat Med 12:235-239 